\title{
Epidemiology of craniosynostosis in Norway
}

\author{
Elin Tønne, MD, 1,2,4 Bernt J. Due-Tønnessen, MD, PhD, ${ }^{3,4}$ Ulrikke Wiig, MD, ,3, \\ Barbro F. Stadheim, MD, ${ }^{2}$ Torstein R. Meling, MD, DPhil, ${ }^{1,3}$ Eirik Helseth, MD, PhD, ${ }^{1,3}$ and \\ Ketil R. Heimdal, MD, PhD2,4
}

\begin{abstract}
${ }^{1}$ Faculty of Medicine, University of Oslo; Departments of ${ }^{2}$ Medical Genetics and ${ }^{3}$ Neurosurgery, and ${ }^{4}$ Norwegian National Unit for Craniofacial Surgery, Oslo University Hospital, Oslo, Norway
\end{abstract}

OBJECTIVE The authors present population-based epidemiological data for craniosynostosis regarding incidence, age at diagnosis, sex differences, and frequency of syndromic and familial cases.

METHODS The prospective registry of the Norwegian National Unit for Craniofacial Surgery was used to retrieve data on all individuals with craniosynostosis treated between 2003 and 2017. The cohort was divided into three 5-year groups based on year of birth: 2003-2007, 2008-2012, and 2013-2017.

\begin{abstract}
RESULTS The authors identified 386 individuals with craniosynostosis. Of these, 328 (85\%) consented to be registered with further information. The incidence increased significantly during the study period and was 5.5 per 10,000 live births $(1 / 1800)$ in the last 5 -year period. The increase was seen almost exclusively in the nonsyndromic group. Syndromic craniosynostosis accounted for $27 \%$ of the cases, and the incidence remained stable throughout the three 5 -year periods. Both syndromic and nonsyndromic craniosynostosis were highly suture specific. There was a male preponderance (male/female ratio 2:1), and males accounted for $75 \%$ of the individuals with midline synostosis. Overall, $9.5 \%$ were index individuals in families with more than one affected member; of these, $73 \%$ were nonsyndromic cases.
\end{abstract}

CONCLUSIONS The incidence of craniosynostosis increased during the study period, and the observed incidence is among the highest reported. The authors attribute this to increasing awareness among healthcare professionals. The number of syndromic cases was high, likely due to a broader definition compared to the majority of earlier reports. The study revealed a high number of familial cases in both syndromic and nonsyndromic craniosynostosis, thus highlighting the importance of genetics as an underlying cause of craniosynostosis.

https://thejns.org/doi/abs/10.3171/2020.1.PEDS2051

KEYWORDS craniosynostosis; epidemiology; incidence; syndromic; nonsyndromic; Norway; craniofacial

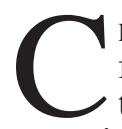

RANIOSYNOSTOSIS is one of the most common malformations in children, usually presenting during the 1 st year of life. ${ }^{1}$ In the healthy population, the metopic suture closes between 6 and 12 months of age, while the other cranial sutures remain open until adulthood. ${ }^{2}$ Premature closure of one or more sutures often results in cranial deformity and compromised intracranial volume. ${ }^{3-6}$ The latter may result in raised intracranial pressure and secondary brain injury. ${ }^{6-9}$

Clinically, craniosynostosis is divided into syndromic and nonsyndromic craniosynostosis. Syndromic craniosynostosis may be characterized by the coexistence of associated malformations, a dysmorphic appearance, or intellectual disability. ${ }^{10}$ The coronal and/or lambdoid sutures are usually affected, often in combination with other sutures. Individuals with nonsyndromic craniosynostosis have no other major findings, and the midline sutures are most commonly affected.

The reported incidence of craniosynostosis varies between 1 in 1600 and 1 in 4000 live births, and it seems to be increasing. ${ }^{11-15}$ Syndromic craniosynostosis constitutes between $12 \%$ and $31 \% \%^{10,11,15,16}$ of all cases. The sagittal suture is the most commonly affected suture, the proportion varying between $41 \%$ and $68 \% .^{10,17-19}$ The male/female ratio ranges from $1.8: 1$ to $4.7: 1,13,15,20-22$ and the proportion of familial craniosynostosis is reported to be between $5.6 \%$ and $14.7 \% .^{14,22,23}$

The epidemiology of craniosynostosis in the literature varies significantly between countries, regions, and medical centers. To our knowledge, the epidemiology of craniosynostosis has not previously been reported from any Scandinavian country. The objective of the current study 
was to present population-based epidemiological data for craniosynostosis with regard to incidence, age at diagnosis, sex differences, and frequency of syndromic and familial cases.

\section{Methods}

Since 2001, all individuals in Norway with suspected craniosynostosis have been referred to the Norwegian National Unit for Craniofacial Surgery at Oslo University Hospital for diagnostic workup, treatment, and follow-up. The registry of the unit is prospective and includes all consenting individuals diagnosed with craniosynostosis.

This population-based study includes individuals with craniosynostosis born between 1/1/2003 and 12/31/2017. From the registry, the following data were extracted on 4/5/2019: date of birth, sex, date of first consultation with a neurosurgeon, affected suture(s), associated findings (other congenital malformations, intellectual disability/ developmental delay, dysmorphic features), genetically confirmed diagnosis, and affected family members.

A clinical assessment of all individuals referred to the Norwegian National Unit for Craniofacial Surgery was performed by one of two pediatric neurosurgeons (B.J.D.T., U.W.). All individuals meeting the criteria for surgical treatment were included in the study. The decision to recommend surgery was based on the risk of the child developing compromised brain growth or progressive deformity of the skull if untreated. Individuals with craniosynostosis who met the criteria, but who were not surgically treated for some reason (diagnosed at an older age, died before surgery, parents resisted), were also included $(n=47)$. The date of the first consultation with the neurosurgeon was defined as age at diagnosis. The affected suture was grouped into metopic, sagittal, unicoronal, or multiple, with the latter group including bicoronal synostosis. All individuals with suspected syndromic craniosynostosis were seen by a clinical geneticist. Syndromic craniosynostosis was defined by the presence of one or more of the following additional findings: 1) malformations (one major or one minor in combination with unusual growth or dysmorphic features), 2) intellectual disability/severe developmental delay, and 3) a genetically confirmed craniosynostosis syndrome. All individuals with syndromic craniosynostosis had been offered genetic analysis, including array comparative genomic hybridization (180K) and high-throughput-sequencing, with a panel of 70 known genes associated with craniosynostosis.

Familial craniosynostosis was defined as having more than one family member with craniosynostosis (first- or second-degree relative).

Based on year of birth, we divided the individuals into three 5-year cohorts (2003-2007, 2008-2012, and 20132017) in order to investigate possible changes regarding the incidence and the age at diagnosis.

\section{Statistical Analysis}

Live birth statistics in the 2003-2017 period were extracted from Statistics Norway. ${ }^{24}$ IBM SPSS Statistics version 25 (IBM Corp.) was used to perform statistical analysis. The OpenEpi Collection of Epidemiological Calcula- tors, version $3.01,{ }^{25}$ was used to calculate chi-square values for the categorical variables. The data are presented as the median and mean values for continuous variables and as the number (\%) for discrete variables. The chi-square test was used to compare categorical variables, and one-way ANOVA was used to compare mean differences in age at diagnosis between the three cohorts, including a Tukey post hoc test. Standard deviations and $p$ values were calculated. Statistical significance was set at $\mathrm{p} \leq 0.05$.

\section{Ethics}

The study was approved by the Norwegian Regional Committees for Medical and Health Research Ethics and by Oslo University Hospital.

\section{Results \\ Incidence}

A total of 386 individuals born between 2003 and 2017 were confirmed to have craniosynostosis requiring surgery. Of these, 328 individuals ( $85 \%$ ) consented to be registered with further information. Norway had a population of 5.3 million in 2017.

The mean annual incidence of craniosynostosis in Norway during the study period was 4.4 cases per 10,000 live births $(1 / 2250)$. In the 2013-2017 cohort, the incidence was 5.5 cases/10,000 live births (1/1800), increasing from 3.3 cases $/ 10,000$ live births $(1 / 3000)$ in the 2003-2007 cohort and 4.3 cases/10,000 live births $(1 / 2300)$ in the 2008-2012 cohort (Fig. 1).

The difference in incidence in the first and last 5-year period constitutes a 70\% increase in registered individuals with craniosynostosis. The chi-square analysis showed this to be a significant difference within all three cohorts (Table 1).

The increasing incidence was almost exclusively seen in nonsyndromic craniosynostosis, while the incidence in syndromic craniosynostosis remained stable over time (Table 1 and Fig. 2). However, within the syndromic group, the incidence of rare genetic syndromes increased significantly. With regard to suture involvement, there was a significant increase in incidence of sagittal craniosynostosis.

\section{Age at Diagnosis}

The median age at diagnosis went down from 204 days in the first 5-year period to 122 days in the last period (Fig. 3 and Table 2). The age at diagnosis went down in both syndromic and nonsyndromic craniosynostosis cases (Fig. 3). The largest reduction in age at diagnosis occurred in individuals with midline craniosynostosis. These individuals were diagnosed at the age of 125 days in the last 5 -year period, compared to 220 days in the first 5-year period. Individuals with syndromic craniosynostosis were diagnosed at a younger age than individuals with nonsyndromic craniosynostosis. The age at diagnosis for individuals with syndromic craniosynostosis was almost equal to the age at diagnosis for individuals with coronal and complex craniosynostosis in the last 5-year period.

Post hoc Tukey analysis showed a significant difference in age at diagnosis between the 2003-2007 and 2013-2017 cohorts only (Table 2). The one-way ANOVA showed a 


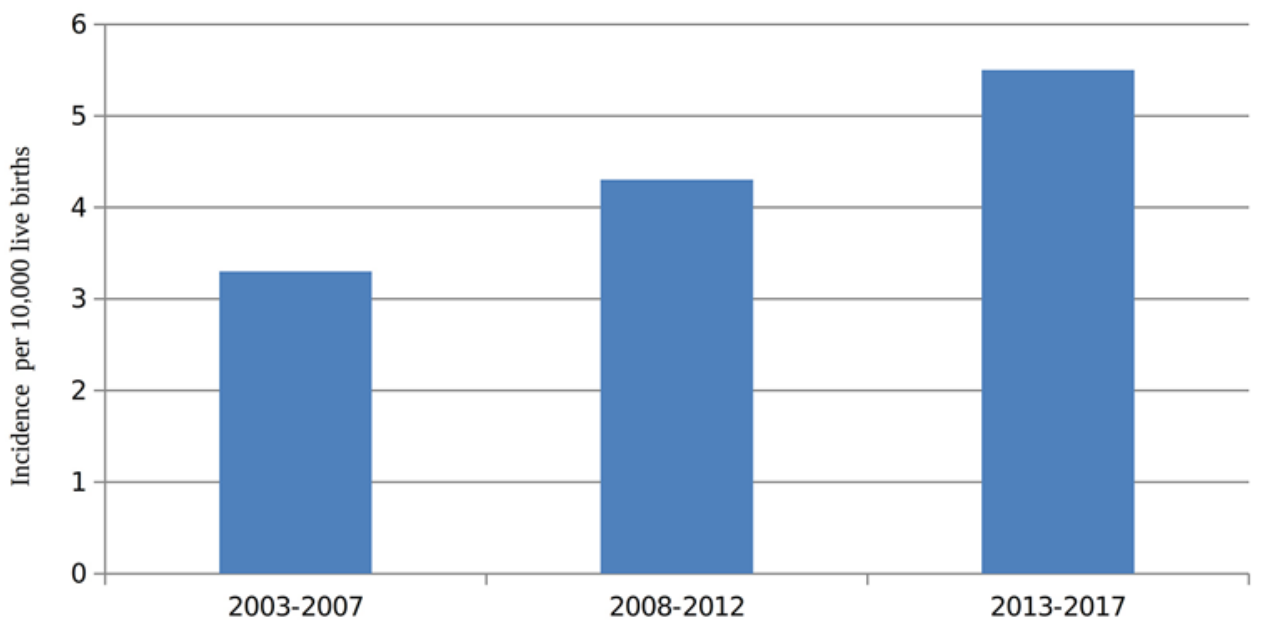

FIG. 1. Period incidence of craniosynostosis in Norway, 2003-2017. Figure is available in color online only.

significant difference between groups $(\mathrm{p}=0.011)$, an Fvalue of 4.5 , and a degree of freedom of 2 .

\section{Syndromic Craniosynostosis}

A total of 89 individuals (27\%) had syndromic craniosynostosis. Of these, 80 individuals accepted routine genetic testing. Of the analyzed individuals, $59(74 \%)$ were confirmed by genetic analysis. Of the individuals with a confirmed genetic diagnosis, 39 individuals (66\%) had one of the common syndromes associated with craniosynostosis (Apert, Crouzon, Muenke, Pfeiffer, or Saethre-Chot- zen syndrome), and 20 (34\%) had a rare genetic syndrome (Table 1).

\section{Affected Suture(s)}

Overall, the sagittal suture was the most commonly affected suture, constituting $49 \%$ of the cases (Table 3). In individuals with syndromic craniosynostosis, multiple affected sutures (including bicoronal synostosis) were the most common finding (43\%). For the remaining syndromic cases, the affected suture was nearly equally distributed among the metopic, sagittal, and unicoronal suture.

TABLE 1. Absolute numbers and period incidence of craniosynostosis per 10,000 live births from 2003 to 2017

\begin{tabular}{|c|c|c|c|c|c|c|c|c|c|}
\hline \multirow[b]{2}{*}{ Craniosynostosis } & \multicolumn{2}{|c|}{$2003-2007$} & \multicolumn{2}{|c|}{ 2008-2012 } & \multicolumn{2}{|c|}{$2013-2017$} & \multicolumn{2}{|c|}{ Total } & \multirow[b]{2}{*}{$p$ Value } \\
\hline & Abs & Incid & Abs & Incid & Abs & Incid & Abs & Incid & \\
\hline Total & 94 & 3.3 & 132 & 4.3 & 160 & 5.5 & 386 & 4.4 & 0.0003 \\
\hline Consenting & 70 & 2.4 & 116 & 3.8 & 142 & 4.9 & 328 & 3.7 & 0.00001 \\
\hline Syndromic & 25 & 0.9 & 31 & 1.0 & 33 & 1.1 & 89 & 1.0 & 0.62 \\
\hline Apert & 3 & 0.1 & 7 & 0.2 & 2 & 0.1 & 12 & 0.1 & 0.20 \\
\hline Muenke & 6 & 0.2 & 3 & 0.1 & 2 & 0.1 & 11 & 0.1 & 0.28 \\
\hline Crouzon/Pfeiffer & 2 & 0.1 & 4 & 0.1 & 1 & 0.03 & 7 & 0.08 & 0.40 \\
\hline Saethre-Chotzen & 3 & 0.1 & 4 & 0.1 & 2 & 0.1 & 9 & 0.1 & 0.75 \\
\hline Rare genetic* & 5 & 0.2 & 3 & 0.1 & 12 & 0.4 & 20 & 0.2 & 0.03 \\
\hline Negative test & 5 & 0.2 & 7 & 0.2 & 9 & 0.3 & 21 & 0.2 & 0.58 \\
\hline Not tested & 1 & 0.03 & 3 & 0.1 & 5 & 0.2 & 9 & 0.1 & 0.27 \\
\hline Nonsyndromic & 45 & 1.2 & 85 & 2.8 & 109 & 3.7 & 239 & 2.7 & 0.00004 \\
\hline \multicolumn{10}{|l|}{ Suture } \\
\hline Sagittal & 25 & 0.9 & 52 & 1.7 & 85 & 2.9 & 162 & 1.8 & 0.0000001 \\
\hline Metopic & 16 & 0.6 & 31 & 1.0 & 31 & 1.1 & 78 & 0.9 & 0.077 \\
\hline Unicoronal & 10 & 0.3 & 13 & 0.4 & 14 & 0.5 & 37 & 0.4 & 0.74 \\
\hline Multiple† & 19 & 0.7 & 20 & 0.7 & 12 & 0.4 & 51 & 0.6 & 0.35 \\
\hline
\end{tabular}

Abs = absolute number; Incid = incidence.

* Rare genetic syndrome, confirmed by genetic analysis.

† Multiple sutures including bicoronal synostosis. 


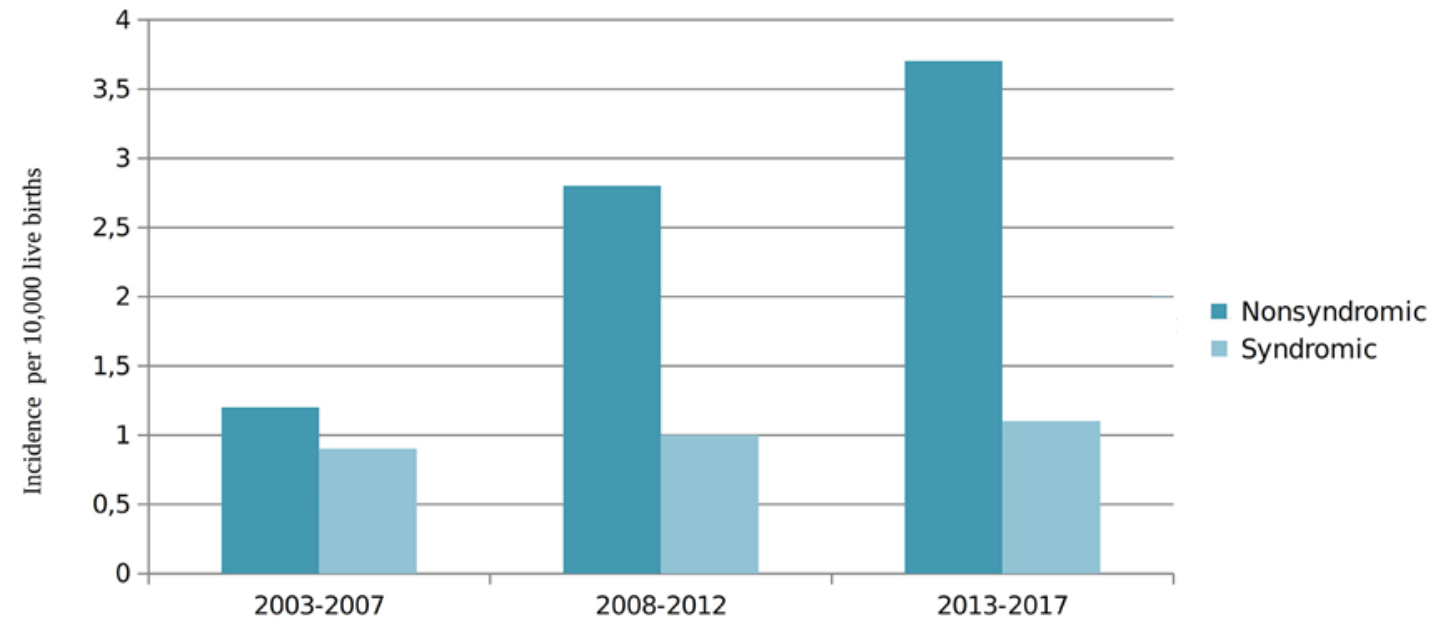

FIG. 2. Period incidence of nonsyndromic and syndromic craniosynostosis, 2003-2017. Figure is available in color online only.

However, in nonsyndromic craniosynostosis the midline sutures, particularly the sagittal suture, were most often affected (Table 3 and Fig. 4).

\section{Sex Differences}

Overall, $67 \%$ of individuals with craniosynostosis were male (Table 4). For metopic and sagittal craniosynostosis, males accounted for $78 \%$ and $73 \%$, respectively. Conversely, for unicoronal synostosis, $62 \%$ were females. In nonsyndromic craniosynostosis, $70 \%$ were male, compared to $58 \%$ in syndromic craniosynostosis.

\section{Familial Cases}

The 328 individuals in our cohort represent 314 different families. Thirty index individuals $(9.5 \%)$ had one affected family member, and $22(73 \%)$ of these had nonsyndromic craniosynostosis.

Registered index individuals in familial craniosynostosis constituted about $10 \%$ of the cases in both syndromic and nonsyndromic craniosynostosis $(10 \%$ and $9.5 \%$, respectively).

\section{Discussion}

In this study, we present population-based epidemiological data on the incidence, age at diagnosis, sex differences, and frequency of syndromic and familial cases of craniosynostosis. We found a high incidence -5.5 per 10,000 live births - in the last 5-year period, increasing from 3.3 per 10,000 live births in the first period. The study not only reveals a high proportion of syndromic cases but also demonstrates that syndromic and nonsyndromic craniosynostosis are highly suture specific.

\section{Incidence}

The increase over the three 5-year periods is significant for individuals with nonsyndromic craniosynostosis and/ or midline synostosis in addition to individuals with rare genetic syndromes.

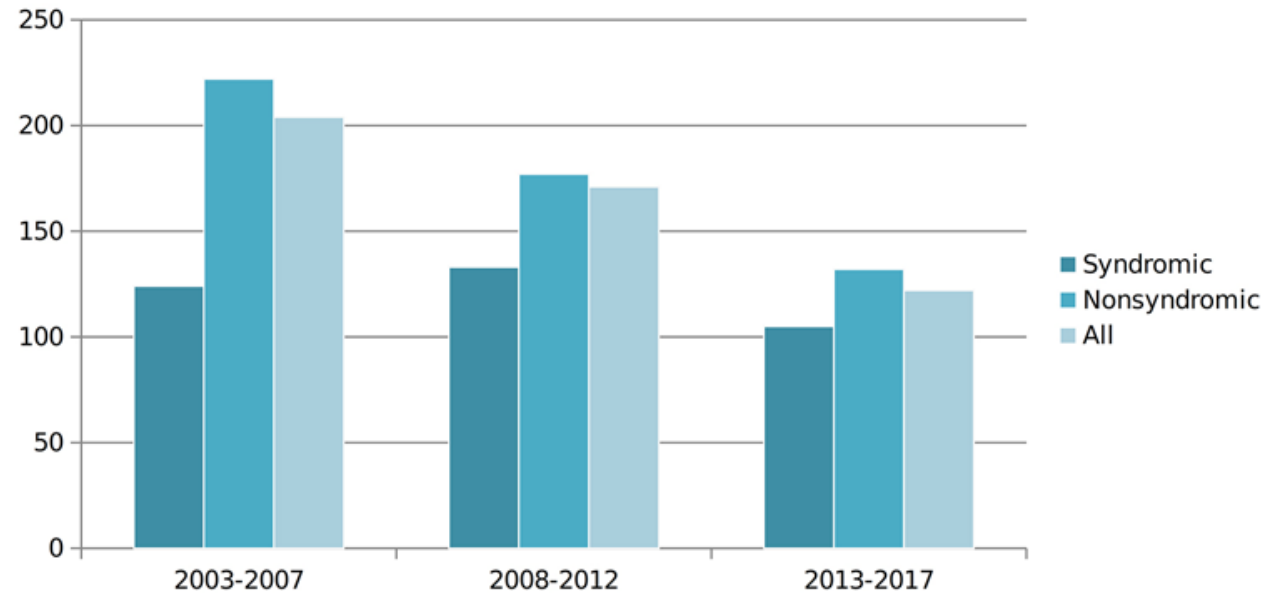

FIG. 3. Median age (in days; y-axis) at diagnosis for syndromic and nonsyndromic craniosynostosis, 2003-2017. Figure is available in color online only. 
TABLE 2. Median age (in days) at diagnosis for nonsyndromic and syndromic craniosynostosis, affected midline and complex suture(s), and total numbers with multiple comparisons

\begin{tabular}{lccccccc}
\hline 5-Yr Period & Syndromic & Nonsyndromic & $\begin{array}{c}\text { Midline } \\
\text { Suture }\end{array}$ & $\begin{array}{c}\text { Complex } \\
\text { Suture* }\end{array}$ & Total & $\begin{array}{c}\text { Multiple } \\
\text { Comparisons } \dagger\end{array}$ & $p$ Value \\
\hline 2003-2007 & $124(866)$ & $222(311)$ & $220(323)$ & $199(775)$ & $204(575)$ & $2008-2012$ & 0.134 \\
& & & & & & $2013-2017$ & 0.008 \\
\hline $2008-2012$ & $133(475)$ & $177(182)$ & $177(267)$ & $148(347)$ & $171(290)$ & $2003-2007$ & 0.134 \\
& & & & & & $2013-2017$ & 0.451 \\
\hline $2013-2017$ & $105(325)$ & $132(160)$ & $125(182)$ & $108(307)$ & $122(210)$ & $2003-2007$ & 0.008 \\
& & & & & & $2008-2012$ & 0.451 \\
\hline Total & $111(571)$ & $170(206)$ & $162(242)$ & $148(520)$ & $162(348)$ & & \\
\hline
\end{tabular}

Values are presented as the median (SD).

* Including uni- and bicoronal synostosis.

† Multiple comparisons between total numbers.

We believe that higher awareness and better diagnostic routines by health professionals are the most likely explanation of the increasing incidence of craniosynostosis. ${ }^{12,26}$ Other factors such as higher paternal age,,$^{14,19}$ higher maternal age, ${ }^{11}$ birth weight, ${ }^{11}$ increasing use of antidepressant medication during pregnancy, ${ }^{27}$ and more have been discussed without any firm associations being established. There may also be an actual increase in the incidence for reasons not yet understood. In particular, nonsyndromic craniosynostosis is most likely the result of multiple factors, both environmental and genetic, the details of which are yet to be elucidated.

The increasing number of reported cases of nonsyndromic midline synostosis has been thoroughly debated. New recommendations that babies should sleep on their back were introduced in Norway in the 1990s, inspired by the international Back to Sleep campaign ${ }^{26}$ in order to prevent sudden infant death syndrome (SIDS). This change in sleeping position brought about a large number of babies seen by health professionals with plagiocephaly, ${ }^{28}$ this again may have contributed to higher awareness of skull development in general and may have contributed to a higher diagnostic rate of craniosynostosis in recent years. ${ }^{26}$
The increasing incidence of rare genetic syndromes is interesting. It may reflect an increasing awareness of rare syndromes with multiorgan affection also manifesting with craniosynostosis, hence an increase in referral. De novo variants in genes involved in growth and development are known to be important mechanisms for these syndromes. ${ }^{10,29-31}$ High paternal age is suggested to increase the risk of de novo variants in general. ${ }^{32,33} \mathrm{We}$ have not accounted for paternal age in our study, but this could be a topic for further investigations. The fact that the incidence of syndromic craniosynostosis was stable over the three time periods in addition to our calculation being based on very low numbers might indicate that this finding is a coincidence that should not be emphasized.

\section{Age at Diagnosis}

The median age at which craniosynostosis was diagnosed went down overall during our study period. In the last 5-year period, the median age was 122 days for all individuals and 105 days for individuals with syndromic craniosynostosis. The younger age at diagnosis was most pronounced in cases of syndromic and/or coronal and complex craniosynostosis. This differs from other studies in which a higher age at diagnosis of complex craniosyn-

TABLE 3. Affected sutures in nonsyndromic and syndromic craniosynostosis presented in absolute numbers and percentages

\begin{tabular}{|c|c|c|c|c|c|c|c|c|c|c|}
\hline \multirow[b]{2}{*}{ Craniosynostosis } & \multicolumn{2}{|c|}{ Sagittal } & \multicolumn{2}{|c|}{ Metopic } & \multicolumn{2}{|c|}{ Unicoronal } & \multicolumn{2}{|c|}{ Multiple* $^{*}$} & \multicolumn{2}{|c|}{ Total } \\
\hline & Abs & $\%$ & Abs & $\%$ & Abs & $\%$ & Abs & $\%$ & Abs & $\%$ \\
\hline Syndromic & 15 & 17 & 18 & 20 & 18 & 20 & 38 & 43 & 89 & 100 \\
\hline Apert & 0 & & 0 & & 1 & & 11 & & 12 & \\
\hline Muenke & 0 & & 0 & & 4 & & 7 & & 11 & \\
\hline Crouzon/Pfeiffer & 1 & & 0 & & 0 & & 6 & & 7 & \\
\hline Saethre-Chotzen & 0 & & 0 & & 6 & & 3 & & 9 & \\
\hline Rare syndrome & 4 & & 5 & & 5 & & 6 & & 20 & \\
\hline Unknown & 10 & & 13 & & 2 & & 5 & & 30 & \\
\hline Nonsyndromic & 147 & 62 & 60 & 25 & 19 & 8 & 13 & 5 & 239 & 100 \\
\hline Total & 162 & 49 & 78 & 24 & 37 & 11 & 51 & 16 & 328 & 100 \\
\hline
\end{tabular}

* Multiple synostosis including bicoronal synostosis. 


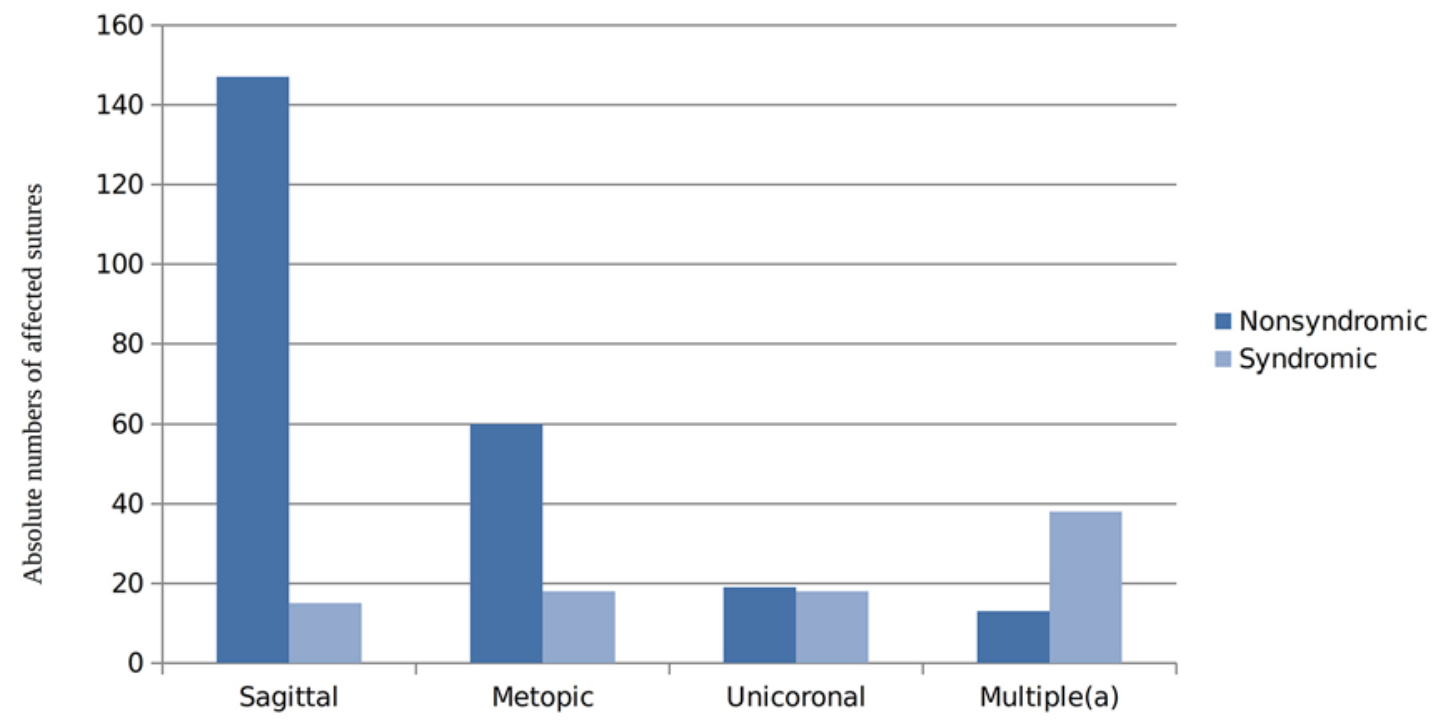

FIG. 4. Absolute numbers of affected suture in syndromic and nonsyndromic craniosynostosis. $a=$ multiple sutures including bicoronal sutures. Figure is available in color online only.

ostosis has been reported. ${ }^{26,28,34}$ The younger age at diagnosis of syndromic and/or complex craniosynostosis might also be explained by greater awareness among health professionals. The fact that nonsyndromic craniosynostosis is diagnosed at a later age may be explained by a higher number of mild cases in which delayed referral occurs due to health professionals' beliefs that there will be improvement in skull shape with time.

\section{Syndromic Cases and Affected Suture(s)}

We report a high proportion of syndromic cases compared to the majority of other published reports. This may be due to previous publications including only the known and common craniosynostosis syndromes (Apert, Crouzon, Muenke, Pfeiffer, and Saethre-Chotzen syndrome) in their syndromic cohorts. There is currently no established consensus for the definition of syndromic craniosynostosis. We believe the grouping of syndromic and nonsyndromic craniosynostosis needs to include a detailed genetic workup. We were able to establish a genetic cause in the majority of the syndromic cases (74\% of the analyzed individuals), supporting the need for a broader definition. We believe this to be important, as a misclassification could

TABLE 4. Affected sutures and sex differences presented in absolute numbers and percentages

\begin{tabular}{lccccccccc}
\hline & \multicolumn{2}{c}{ Female } & & \multicolumn{2}{c}{ Male } & & \multicolumn{2}{c}{ Total } \\
\cline { 2 - 3 } \cline { 9 - 10 } Affected Suture & Abs & $\%$ & & Abs & $\%$ & & Abs & $\%$ \\
\hline Sagittal & 44 & 27 & & 118 & 73 & & 162 & 100 \\
\hline Metopic & 17 & 22 & & 61 & 78 & & 78 & 100 \\
\hline Unicoronal & 23 & 62 & & 14 & 38 & & 37 & 100 \\
\hline Multiple* & 24 & 47 & & 27 & 53 & & 51 & 100 \\
\hline Total & 108 & 33 & & 220 & 67 & & 328 & 100 \\
\hline
\end{tabular}

${ }^{*}$ Multiple sutures including bicoronal sutures. potentially impact and bias the research on craniosynostosis beyond epidemiology. This could be exemplified by studies on neuropsychological outcomes of individuals with nonsyndromic craniosynostosis, where a presumably nonsyndromic cohort actually includes a number of syndromic cases, thus biasing the results toward a higher proportion of neuropsychological deficits.

We found a higher proportion of sagittal and metopic suture involvement in our nonsyndromic cohort than has previously been reported in the literature. In addition, we found a high proportion of bicoronal and multiple-suture involvement in syndromic craniosynostosis, with a low proportion of sagittal synostosis in this group. This emphasizes the argument that syndromic and nonsyndromic craniosynostosis are highly suture specific, probably related to different embryological mechanisms and signaling pathways in part regulated by genetics. ${ }^{1,20}$

\section{Sex Differences}

Many reports have shown a male preponderance in midline synostosis, and our data support this finding. This does not extend to unicoronal synostosis, in which the majority of cases are female, supporting earlier conclusions that the overall male predominance of craniosynostosis does not include the coronal suture..$^{14,20}$ The cause for this sex bias is still to be established. Whether male dominance is partly due to genetic variants on the $\mathrm{X}$ chromosome or the cause of circulating androgens in early craniofacial development ${ }^{35}$ is yet to be established.

\section{Familial Cases}

The high number of familial cases in nonsyndromic craniosynostosis suggests a genetic cause, in addition to the more established genetic involvement in syndromic craniosynostosis. The genes involved in nonsyndromic craniosynostosis are in the beginning of their discovery, and inheritance seems to be complex. ${ }^{36}$ 


\section{Study Strengths and Limitations}

One of the major strengths of this study is that the epidemiological data are population based and collected prospectively. Previous reports are mainly based on data from single hospitals or regions (with a few exceptions ${ }^{13}$ ), and this might explain the broad range found with regard to the incidence, proportion of syndromic to nonsyndromic cases, affected suture(s), sex distribution, and frequency of familial cases. All individuals with suspected syndromic or familial craniosynostosis were seen by one of the team's clinical geneticists, thus allowing more accurate figures for syndromic and familial cases. We believe Norway to be a suitable country for studying the epidemiology of craniosynostosis because of the equal-access healthcare system that ensures a high inclusion rate and because of the organization of the unit in a centralized multidisciplinary team diagnosing and treating all individuals with craniosynostosis.

The main limitation in any study of the incidence of craniosynostosis is the lack of objective diagnostic criteria. All published classification systems have an element of subjective expert opinion. Our series is based on diagnosis by the same experienced pediatric neurosurgeons. Mild cases/partial synostoses not requiring surgery were excluded. A full radiological workup was not routinely performed. Partly fused single-suture cases with minimal stigmata would therefore not have been registered. We have included cases judged by the unit's experienced pediatric neurosurgeons to be surgical candidates but in which surgery was deferred for different reasons. Thus, our series may not be directly comparable to others.

Another limitation of the study is that we cannot be entirely sure that all individuals in need of surgery were referred to the National Unit for Craniofacial Surgery as recommended and may have been treated at a local/regional hospital. We made an inquiry to the Norwegian Directorate of Health, which in turn provided summary statistics of all individuals surgically treated in 2008-2016. This revealed a small number of individuals who had undergone relevant surgery at one of the regional hospitals outside Oslo. Unfortunately, we do not have all relevant information regarding these patients, as the data provided are anonymous, do not include age, and are only linked to the procedure performed. Furthermore, we believe that some of the individuals operated on elsewhere were included in our database, having been referred to the National Unit for further treatment and follow-up. In effect, this means that the overall incidence may be slightly higher than presented in this series. The surgeries performed outside Oslo are equally distributed over the study period, and therefore the impact on the increase in incidence is negligible.

With the exception of overall incidence, we have included only consenting individuals $(85 \%)$ in the study. This could potentially bias the results. However, to the best of our knowledge, the nonconsenting individuals were distributed equally for all parameters.

We also included as a limitation the possibility that there may be children born in the last few years of the study yet to be diagnosed. However, we expect this number to be low, as most children are diagnosed by 1 year of age.

\section{Conclusions}

This population-based epidemiological study confirms previous reports of an increasing incidence of craniosynostosis. In addition, we present one of the highest incidence rates reported in the literature. The increase is primarily found in nonsyndromic, midline craniosynostosis. Children are diagnosed at a younger age in the latter cohort, and this is more pronounced for individuals with syndromic or complex craniosynostosis. The high number of syndromic cases presented in this study is probably related to a broader definition when compared to other studies. We demonstrate that syndromic craniosynostosis is highly suture specific. The study revealed a high occurrence of familial cases in both syndromic and nonsyndromic craniosynostosis, further emphasizing the importance of genetics in craniosynostosis.

\section{Acknowledgments}

We would like to thank Elisabeth Elgesem and Grete Furseth for excellent secretarial assistance.

This work is supported by the Norwegian National Advisory Unit on Rare Disorders with a grant covering salary for 1 year to Elin Tønne.

\section{References}

1. Twigg SR, Wilkie AO. A genetic-pathophysiological framework for craniosynostosis. Am J Hum Genet. 2015;97(3):359377.

2. Weinzweig J, Kirschner RE, Farley A, et al. Metopic synostosis: defining the temporal sequence of normal suture fusion and differentiating it from synostosis on the basis of computed tomography images. Plast Reconstr Surg. 2003;112(5):1211-1218.

3. Eide PK, Helseth E, Due-Tønnessen B, Lundar T. Assessment of continuous intracranial pressure recordings in childhood craniosynostosis. Pediatr Neurosurg. 2002;37(6):310-320.

4. Judy BF, Swanson JW, Yang W, et al. Intraoperative intracranial pressure monitoring in the pediatric craniosynostosis population. J Neurosurg Pediatr. 2018;22(5):475-480.

5. Proctor MR, Meara JG. A review of the management of single-suture craniosynostosis, past, present, and future. $J$ Neurosurg Pediatr. 2019;24(6):622-631.

6. Thompson DN, Harkness W, Jones BM, Hayward RD. Aetiology of herniation of the hindbrain in craniosynostosis. An investigation incorporating intracranial pressure monitoring and magnetic resonance imaging. Pediatr Neurosurg. 1997;26(6):288-295.

7. Nguyen JQN, Resnick CM, Chang YH, et al. Impact of obstructive sleep apnea on optic nerve function in patients with craniosynostosis and recurrent intracranial hypertension. Am J Ophthalmol. 2019;207:356-362.

8. Renier D, Sainte-Rose C, Marchac D, Hirsch JF. Intracranial pressure in craniostenosis. J Neurosurg. 1982;57(3):370-377.

9. Thompson DN, Harkness W, Jones B, et al. Subdural intracranial pressure monitoring in craniosynostosis: its role in surgical management. Childs Nerv Syst. 1995;11(5):269-275.

10. Wilkie AOM, Johnson D, Wall SA. Clinical genetics of craniosynostosis. Curr Opin Pediatr. 2017;29(6):622-628.

11. Boulet SL, Rasmussen SA, Honein MA. A population-based study of craniosynostosis in metropolitan Atlanta, 19892003. Am J Med Genet A. 2008;146A(8):984-991.

12. Cornelissen M, Ottelander Bd, Rizopoulos D, et al. Increase of prevalence of craniosynostosis. J Craniomaxillofac Surg. 2016;44(9):1273-1279.

13. Kweldam CF, van der Vlugt JJ, van der Meulen JJ. The inci- 
dence of craniosynostosis in the Netherlands, 1997-2007. $J$ Plast Reconstr Aesthet Surg. 2011;64(5):583-588.

14. Lajeunie E, Le Merrer M, Bonaïti-Pellie C, et al. Genetic study of nonsyndromic coronal craniosynostosis. Am J Med Genet. 1995;55(4):500-504.

15. Neusel C, Class D, Eckert AW, et al. Multicentre approach to epidemiological aspects of craniosynostosis in Germany. Br J Oral Maxillofac Surg. 2018;56(9):881-886.

16. Garrocho-Rangel A, Manriquez-Olmos L, Flores-Velazquez $\mathrm{J}$, et al. Non-syndromic craniosynostosis in children: scoping review. Med Oral Patol Oral Cir Bucal. 2018;23(4):e421e428.

17. Hunter AG, Rudd NL. Craniosynostosis. I. Sagittal synostosis: its genetics and associated clinical findings in 214 patients who lacked involvement of the coronal suture(s). Teratology. 1976;14(2):185-193.

18. Selber J, Reid RR, Chike-Obi CJ, et al. The changing epidemiologic spectrum of single-suture synostoses. Plast Reconstr Surg. 2008;122(2):527-533.

19. van der Meulen J, van der Hulst R, van Adrichem L, et al. The increase of metopic synostosis: a pan-European observation. J Craniofac Surg. 2009;20(2):283-286.

20. Greenwood J, Flodman P, Osann K, et al. Familial incidence and associated symptoms in a population of individuals with nonsyndromic craniosynostosis. Genet Med. 2014;16(4):302310 .

21. Lajeunie E, Le Merrer M, Bonaïti-Pellie C, et al. Genetic study of scaphocephaly. Am J Med Genet. 1996;62(3):282285.

22. Lajeunie E, Le Merrer M, Marchac D, Renier D. Syndromal and nonsyndromal primary trigonocephaly: analysis of a series of 237 patients. Am J Med Genet. 1998;75(2):211-215.

23. Kalantar-Hormozi H, Abbaszadeh-Kasbi A, Sharifi G, et al. Incidence of familial craniosynostosis among patients with nonsyndromic craniosynostosis. J Craniofac Surg. 2019;30(6):e514-e517.

24. Statistics Norway (SSB). Population: Births. Updated March 7, 2019. Accessed February 13, 2020. https://www.ssb.no/ befolkning/statistikker/fodte

25. Dean AG, Sullivan KM, Soe MM. R by C table. OpenEpi: Open Source Epidemiologic Statistics for Public Health. Accessed February 13, 2020. http://openepi.com/RbyC/RbyC. htm

26. Branch LG, Kesty K, Krebs E, et al. Deformational plagiocephaly and craniosynostosis: trends in diagnosis and treatment after the "back to sleep" campaign. J Craniofac Surg. 2015;26(1):147-150.

27. Bérard A, Zhao JP, Sheehy O. Antidepressant use during pregnancy and the risk of major congenital malformations in a cohort of depressed pregnant women: an updated analysis of the Quebec Pregnancy Cohort. BMJ Open. 2017;7(1):e013372.
28. Argenta LC, David LR, Wilson JA, Bell WO. An increase in infant cranial deformity with supine sleeping position. J Craniofac Surg. 1996;7(1):5-11.

29. Heuzé Y, Holmes G, Peter I, et al. Closing the gap: genetic and genomic continuum from syndromic to nonsyndromic craniosynostoses. Curr Genet Med Rep. 2014;2(3):135-145.

30. Miller KA, Twigg SR, McGowan SJ, et al. Diagnostic value of exome and whole genome sequencing in craniosynostosis J Med Genet. 2017;54(4):260-268.

31. Xu Y, Sun S, Li N, et al. Identification and analysis of the genetic causes in nine unrelated probands with syndromic craniosynostosis. Gene. 2018;641:144-150.

32. Simard M, Laprise C, Girard SL. Impact of paternal age at conception on human health. Clin Chem. 2019;65(1):146-152.

33. Taylor JL, Debost JPG, Morton SU, et al. Paternal-age-related de novo mutations and risk for five disorders. Nat Commun. 2019;10(1):3043.

34. Seruya M, Oh AK, Boyajian MJ, et al. Age at initial consultation for craniosynostosis: comparison across different patient characteristics. J Craniofac Surg. 2013;24(1):96-98.

35. Lin IC, Slemp AE, Hwang C, et al. Immunolocalization of androgen receptor in the developing craniofacial skeleton. $J$ Craniofac Surg. 2004;15(6):922-929.

36. Timberlake AT, Persing JA. Genetics of nonsyndromic craniosynostosis. Plast Reconstr Surg. 2018;141(6):1508-1516.

\section{Disclosures}

The authors report no conflict of interest concerning the materials or methods used in this study or the findings specified in this paper.

\section{Author Contributions}

Conception and design: Tønne, Due-Tønnessen, Helseth, Heimdal. Acquisition of data: Tønne, Due-Tønnessen, Wiig, Stadheim, Heimdal. Analysis and interpretation of data: Tønne, Due-Tønnessen, Meling, Helseth, Heimdal. Drafting the article: Tønne. Critically revising the article: Due-Tønnessen, Wiig, Stadheim, Meling, Helseth, Heimdal. Statistical analysis: Tønne. Study supervision: Meling, Helseth, Heimdal.

\section{Supplemental Information Current Affiliations}

Dr. Meling: Department of Neurosurgery, Geneva University Hospitals, Geneva, Switzerland.

\section{Correspondence}

Elin Tønne: Oslo University Hospital, Oslo, Norway. elin.tonne@ gmail.com. 